\title{
Trolline Ameliorates Liver Fibrosis by Inhibiting the NF-кB Pathway, Promoting HSC Apoptosis and Suppressing Autophagy
}

\author{
Facheng Bai Quanfang Huang Jinlan Nie Shengjuan Lu Chunyuan Lu \\ Xunshuai Zhu Yuxin Wang Lang Zhuo Zhongpeng Lu Xing Lin \\ Guangxi Medical University, Nanning, China
}

Key Words

Trolline $\cdot$ Hepatic fibrosis $・$ NF-KB pathway $•$ Autophagy

\begin{abstract}
Background/Aims: Previous studies have shown that trolline possesses various forms of pharmacological activity, including antibacterial and antiviral potency. The present paper addressed the putative hepatoprotective effects of trolline. Methods: Rats received $2 \mathrm{ml} / \mathrm{kg}$ $\mathrm{CCl}_{4}$ (mixed 1:1 in peanut oil) intragastrically twice a week for 8 weeks to induce hepatic fibrosis. The animals were then treated with trolline for additional 4 weeks. Liver pathology and collagen accumulation were observed by hematoxylin-eosin and Masson's trichrome staining, respectively. Serum transaminase activity and collagen-related indicator level were determined by commercially available kits. NF-kB pathway activation was also examined. Moreover, the effects of trolline on hepatic stellate cell (HSC-T6) apoptosis, mitochondrial membrane potential (MMP), and autophagy were assessed. Results: Trolline significantly alleviated $\mathrm{CCl}_{4}$-induced liver injury and notably reduced the accumulation of collagen in liver tissues. Trolline treatment also markedly decreased inflammatory cytokines levels by inhibiting the NF-KB pathway. Trolline strongly inhibited HSC-T6 activation and notably induced cell apoptosis by modulating the $\mathrm{Bax} / \mathrm{Bcl}-2$ ratio, caspase activity, and MMP. Moreover, trolline significantly inhibited HSC-T6 autophagy, as evidenced by the decrease in the formation of autophagic vacuoles and the number of autophagosomes, by regulating the expression levles of LC3, Beclin-1, P62, Atg 5 and 7. Conclusion: Our study demonstrates that trolline ameliorates liver fibrosis, possibly by inhibiting the NF-KB pathway, promoting HSCs apoptosis and suppressing autophagy.




\section{Introduction}

Hepatic fibrosis, characterized by excessive formation of the extracellular matrix (ECM), is the essential pathophysiologic consequence of chronic hepatic injury [1, 2]. Hepatic stellate cells (HSCs) are the main source of the ECM, and their activation is a pivotal event in hepatic fibrogenesis $[3,4]$. Thus, enhancing the apoptosis of activated HSCs is of great importance to the treatment of liver fibrosis [5]. In addition to the activation of HSCs, fibrosis-inducing events also lead to the recruitment of inflammatory and Kupfer cells and the subsequent release of reactive oxygen species and cytokines. The transcription factor nuclear factorkappa B (NF- $\mathrm{B}$ ) is well known for its involvement in inflammatory responses. Regulation of cell death and inflammation by NF- $\kappa \mathrm{B}$ not only emphasizes the role of this transcription factor in the progression of liver diseases but highlights the mechanistic links among liver injury, inflammation, fibrosis, and hepatocellular carcinoma. Previous studies have confirmed that $\mathrm{NF}-\kappa \mathrm{B}$ inhibition is a potential mechanism for the induction of HSC apoptosis [6]. Hence, this pathway presents a critical target to ameliorate inflammation and liver injury to lessen the severity of liver fibrosis [7].

Autophagy is a metabolic process in which cells degrade their own components under exogenetic stimulation, such as energy restriction, stress, or inflammation. In this condition, autophagy allows cells to degrade damaged organelles, such as non-functional mitochondria, thus facilitating cell survival [8]. Autophagy is involved in the degradation of long-lived organelles and can promote the survival and activation of HSCs [9]. Moreover, it induces the release of lipid droplets from HSCs and promotes the fibrogenesis of activated HSCs [10], suggesting that autophagy is closely associated with liver fibrosis.

Trolline is an isoquinoline alkaloid that has been isolated from the flowers of Trollius chinensis Bunge (Ranunculaceae) [11]. It exhibits antibacterial activity against respiratory bacteria such as Staphylococcus aureus, Streptococcus pneumoniae and Klebsiella pneumoniae, and antiviral activity against influenza virus A and B $[12,13]$. Recently, our pilot experiment showed that trolline significantly induced HSC apoptosis. Thus, we hypothesized that it may play a potential role in the treatment of liver fibrosis. In the present study, trolline was synthesized and its protective effect on hepatic fibrosis was investigated both in vivo and in vitro.

\section{Materials and Methods}

\section{Synthesis of trolline}

Trolline was synthesized as previously described [13], and the synthetic route and reaction conditions are presented in Fig. 1.

\section{Animals and treatment}

Male SD rats (200 $\pm 20 \mathrm{~g}$ ) were obtained from Guangxi Medical University Experimental Animal Center (Guangxi, China). This study was conducted according to the protocol approved by the Guangxi Medical University Institutional Ethical Committee. In brief, 60 rats were randomly divided into four groups 15 rats per group) including normal control group, trolline control group, $\mathrm{CCl}_{4}$ model group and trolline -treated group. The rats in the last two groups received $2 \mathrm{ml} / \mathrm{kg} \mathrm{CCl}_{4}$ (mixed 1:1 in peanut oil) intragastrically twice a week for 12 weeks, while the animals in the first two groups received equivalent amounts of peanut oil. Meanwhile, during weeks 8 to 12 , rats in the trolline-treated group were intraperitoneally administered with $0.5 \mathrm{mg} / \mathrm{kg}$ trolline once a day; while animals in the $\mathrm{CCl}_{4}$ model group received equivalent amounts of normal saline. In addition, rats in the normal control and trolline control groups were intraperitoneally administered with equivalent amounts of normal saline and $0.5 \mathrm{mg} / \mathrm{kg}$ trolline, respectively, from weeks 8 to 12. At the end of treatment, all animals were sacrificed and blood and liver samples were obtained for further examination. 
Fig. 1. The synthetic route and reaction conditions of trolline. Reagents and conditions: (a) oxalyl chloride, $\mathrm{N}, \mathrm{N}-$ dimethylformamide, dichloromethane, room temperature, $4 \mathrm{~h}$; (b) triethylamine, $\mathrm{CH}_{2} \mathrm{Cl}_{2}$, room temperature, 12 h; (c) phosphorus oxychloride, dichloromethane, room temperature, $5 \mathrm{~h} ; \mathrm{NaBH}_{4}$, $\mathrm{CH}_{3} \mathrm{OH}$, room temperature, 2 $\mathrm{h}$; (d) $\mathrm{BBr}_{3^{\prime}}$ dichloromethane, room temperature, $2 \mathrm{~h}$.

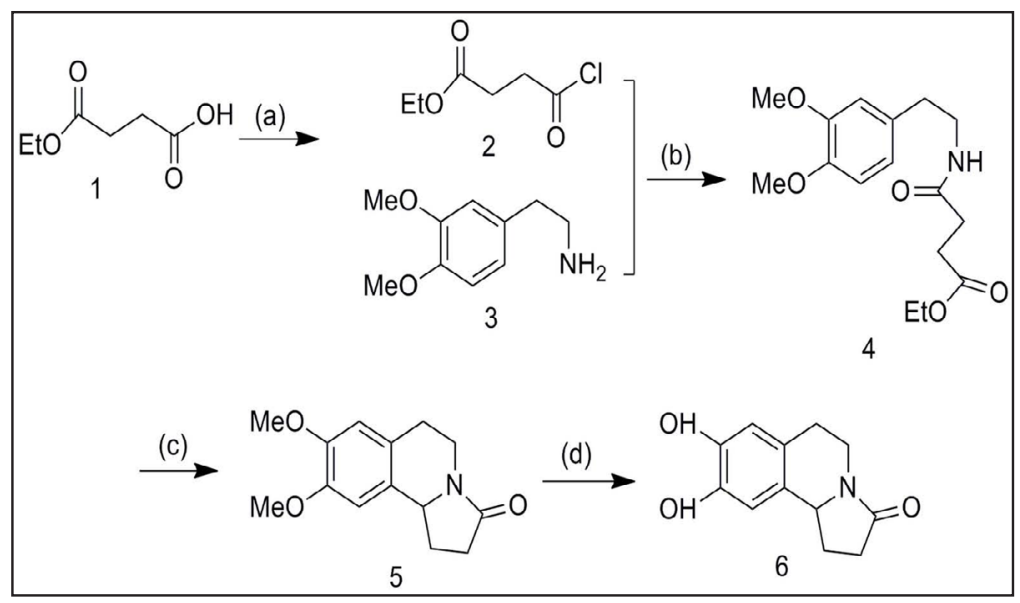

Histological examination

Liver fragments were fixed in $10 \%$ formalin and embedded in paraffin. Five $\mu \mathrm{m}$ sections were then cut and mounted on glass slides. The pathological damage was observed by hematoxylin-eosin (H\&E) staining and the collagen deposition was observed by Masson's trichrome staining as described in our previous work [14].

\section{Serological tests}

Serum levels of alanine transaminase (ALT), aspartate transaminase (AST), alkaline phosphatase (ALP) and c-glutamyltransferase (GGT) were measured by using commercially available kits (Nanjing Jiancheng Bioengineering Research Institute, Nanjing, China); plasma TNF- $\alpha$ and IL-1 $\beta$ were detected using an enzyme-linked immunosorbent assay kit (Beijing Yonghui Biological Technology, Beijing, China), according to the manufacturer's instructions.

\section{Determination of collagen-related indicators}

The collagen-related indicators including hydroxyproline (Hyp), type III precollagen (PCIII), laminin (LN) and hyaluronic acid (HA) were determined by radioimmunoassay (RIA) using commercially available kits (Beijing Furui Bioengi- neering Research Company, Beijing, China) according to the manufacturer's instructions.

\section{Determination of NF- $\kappa B$ pathway activation}

Total hepatic protein was extracted from liver tissues with radioimmunoprecipitation buffer containing a protease inhibitor cocktail (Sigma-Aldrich). Western blot was performed to assess the NF- $\mathrm{\kappa B}$ pathway as described in our previous work [15]. The antibodies, including NF-KB-p65 (p65), phospho-NF-kB-p65 (pp65), IкB $\alpha$, phospho-IкB $\alpha$ (p-IкB $\alpha$ ) and GAPDH, were provided by Santa Cruz, USA.

\section{Cell culture and cytotoxicity test}

HSC-T6 cells were provided by Shanghai Meixuan Biological Science and Technology Ltd. (Shanghai, China) and were cultured in Dulbecco's modified eagle medium (DMEM) with $10 \% \mathrm{FBS}, 100 \mathrm{IU} / \mathrm{ml}$ penicillinstreptomycin and $1 \%$ glutamine in a humidified incubator under $5 \% \mathrm{CO}_{2}$ at $37^{\circ} \mathrm{C}$. Cells were seeded in 96 well plates $\left(3 \times 10^{5}\right.$ cells per well $)$ for $24 \mathrm{~h}$, and followed by treatment with various concentrations of trolline for $24 \mathrm{~h}$. Finally, $100 \mu \mathrm{L}$ MTT ( $5 \mathrm{mg} / \mathrm{mL}$ ) was added to each well and incubated for $4 \mathrm{~h}$. The absorbance was measured at $570 \mathrm{~nm}$ using a microplate reader (Bio-Tek, USA). Survival $(\%)=100 \times\left(1-\mathrm{OD}_{\text {drug }} / \mathrm{OD}_{\text {control }}\right) \%$.

\section{Detection of $\alpha$-SMA and collagen I expression levels}

The expression levels of $\alpha$-SMA and collagen I in HSC-T6 cells were detected using Western blot assay as described in our previous work [2]. Briefly, HSC-T6 cells were seeded in 6-well plates $\left(3 \times 10^{5}\right.$ cells per well) overnight, then divided into four groups including normal control group (cells were treated with equal amounts of culture medium), trolline group (cells were treated with $10 \mu \mathrm{M}$ trolline), PDGF-BB group (cells were treated with $10 \mathrm{nM}$ PDGF-BB), and trolline + PDGF-BB group (cells were treated with10 $\mu \mathrm{M}$ trolline plus $10 \mathrm{nM}$ PDGF-BB). Cells were treated with drugs (dissolved in $0.1 \%$ DMSO) for $24 \mathrm{~h}$. The whole cell proteins were prepared by using RIPA buffer (Thermo Fischer Scientific, Inc., Waltham, MA). The antibodies 


\section{Cellular Physiology Cell Physiol Biochem 2017;44:436-446 \\ \begin{tabular}{l|l|l} 
DOI: 10.1159/000485009 & $\begin{array}{l}\text { O 2017 The Author(s). Published by S. Karger AG, Basel } \\
\text { www.karger.com/cpb }\end{array}$
\end{tabular}}

Bai et al.: Trolline Ameliorates Liver Fibrosis

including $\alpha$-SMA and collagen I were obtained from Cell Signaling Technology, and GAPDH (Santa Cruz Biotechnology) was used as an internal control.

\section{Apoptosis analysis}

HSC-T6 cells were seeded in 6-well plates ( $3 \times 10^{5}$ cells per well) overnight, then treated with $2.5,5$ and $10 \mu \mathrm{M}$ trolline (dissolved in 0.1\% DMSO) for $24 \mathrm{~h}$. The Annexin-V-FITC Apoptosis Detection Kit (Bestbio, China) was used to evaluate cell apoptosis according to the manufacturer's instruction. The samples were analyzed within $1 \mathrm{~h}$ by FACS using CellQuest software. The experiments were repeated three times.

\section{Detection of apoptosis-related protein expression and caspase activity}

HSC-T6 cells were plated in 6-well plates $\left(3 \times 10^{5}\right.$ cells per well) overnight and treated with drugs for $24 \mathrm{~h}$. The cells were washed twice with cold PBS, resuspended in lysis buffer and centrifuged at 15, $000 \times \mathrm{g}$ at $4{ }^{\circ} \mathrm{C}$ for $15 \mathrm{~min}$. Supernatants were collected and protein concentrations were measured using a Protein Assay Kit (Pierce, Rockford, IL, USA). The apoptosis-related proteins including Bcl-2 and Bax were detected by Western blotting. Caspase-3 and -8 were detected using commercially available kits (BioVision Research Products, CA, USA).

\section{Detection of mitochondrial membrane potential}

Mitochondrial membrane potential (MMP) is an indicator of mitochondrial function. In the present study, HSC-T6 cells were seeded in 96 well-plates $\left(3 \times 10^{5}\right.$ cells per well) overnight and treated with drugs for $24 \mathrm{~h}$. MMP was measured using the lipophilic cationic dye JC-1 (5, 5',6, 6'-tetrachloro -1, 1',3, 3'tetraethylbenzimi- dazolylcarbocyanine iodide), as described in our previous study [16].

\section{Detection of autophagic vacuoles}

Autophagic vacuoles were detected by monodansylcadaverine (MDC) staining as previously described [17]. In brief, HSC-T6 cells were seeded in 6-well plates ( $3 \times 10^{5}$ cells per well) and divided into five groups including normal control group, trolline control group (cells were incubated with $10 \mu \mathrm{M}$ trolline), autophagy model group, 3-MA group (cells were treated with $5 \mathrm{mM} 3$-methyladenine) and trolline-treated group (cells were treated with $10 \mu \mathrm{M}$ trolline). The cells were incubated with drugs (dissolved in $0.1 \%$ DMSO) for 24 h. Cells in the last three groups were serum starved for $24 \mathrm{~h}$ in $0.5 \%$ FBS to induce autophagy. Thereafter, cells were incubated with $0.05 \mathrm{mM}$ monodansyl cadaverine in PBS at $37^{\circ} \mathrm{C}$ for $10 \mathrm{~min}$. The cells were than washed 4 times with PBS and observed under a fluorescence microscope at an excitation wavelength of 380 $\mathrm{nm}$ and emission wavelength of $525 \mathrm{~nm}$.

\section{Transmission electron microscopy}

HSC-T6 cells were seeded in 6 well-plates $\left(3 \times 10^{5}\right.$ cells per well) overnight, then treated with drugs for $24 \mathrm{~h}$. Cells were sequentially harvested for morphological examination using transmission electron microscopy as previously described [18].

\section{RT-PCR assay}

Total RNA was extracted by TRIzol reagent (Life Technologies, Inc.) according to the manufacturer's instructions, and RT-PCR assay was performed as described in our previous study [19]. The primers used in this study were as follows: NF-kB: 5'-GTT ATC GTT CAG TTG GTC ACA-3' and 5'-ATA TGC CGT CCT CAC AGT-3'; Atg5: 5'-GGC CAT CAA TCG GAA ACT CAT-3' and 5'-CAG CCA CAG GAC GAA ACA GC-3'; Atg7: 5'-GGC TCA TCG CTT TTT GCC A AC ATC-3' and 5'-GCA CTG CTG GTC CAA GGT CCG-3'; GAPDH: 5'-CGC CTG CTT CAC CAC CTT-3' and 5'-CCT GCC GCC TGG AGA AA-3'.

\section{Autophagy-related proteins expression}

Whole cell proteins were prepared using RIPA buffer (Thermo Fischer Scientific, Inc., Waltham, MA). Western blot was carried out as described in our previous work [2]. The antibodies used in the present study were: Atg5, Atg7, Beclin-1, LC3 I/II and P62 (1:500) (Cell Signaling Technology). The detected proteins were normalized to GAPDH (Santa Cruz Biotechnology) or the respective total protein, as appropriate.

\section{Statistical analysis}

Statistical analysis was carried out using the Software of SPSS software (Ver.11.5) as described in our previous study [2]. Differences between the groups were assessed using a one-way analysis of variance (ANOVA) with a Tukey's test for post hoc multiple comparisons. The data are presented as the means \pm SD. A $p$-value $<0.05$ was considered statistically significant.

\section{KARGER}




\section{Cellular Physiology Cell Physiol Biochem 2017;44:436-446 \begin{tabular}{l|l|l} 
and Biochemistry $10.1159 / 000485009$ & $\begin{array}{l}\text { O 2017 The Author(s). Published by S. Karger AG, Basel } \\
\text { www.karger.com/cpb }\end{array}$
\end{tabular}}

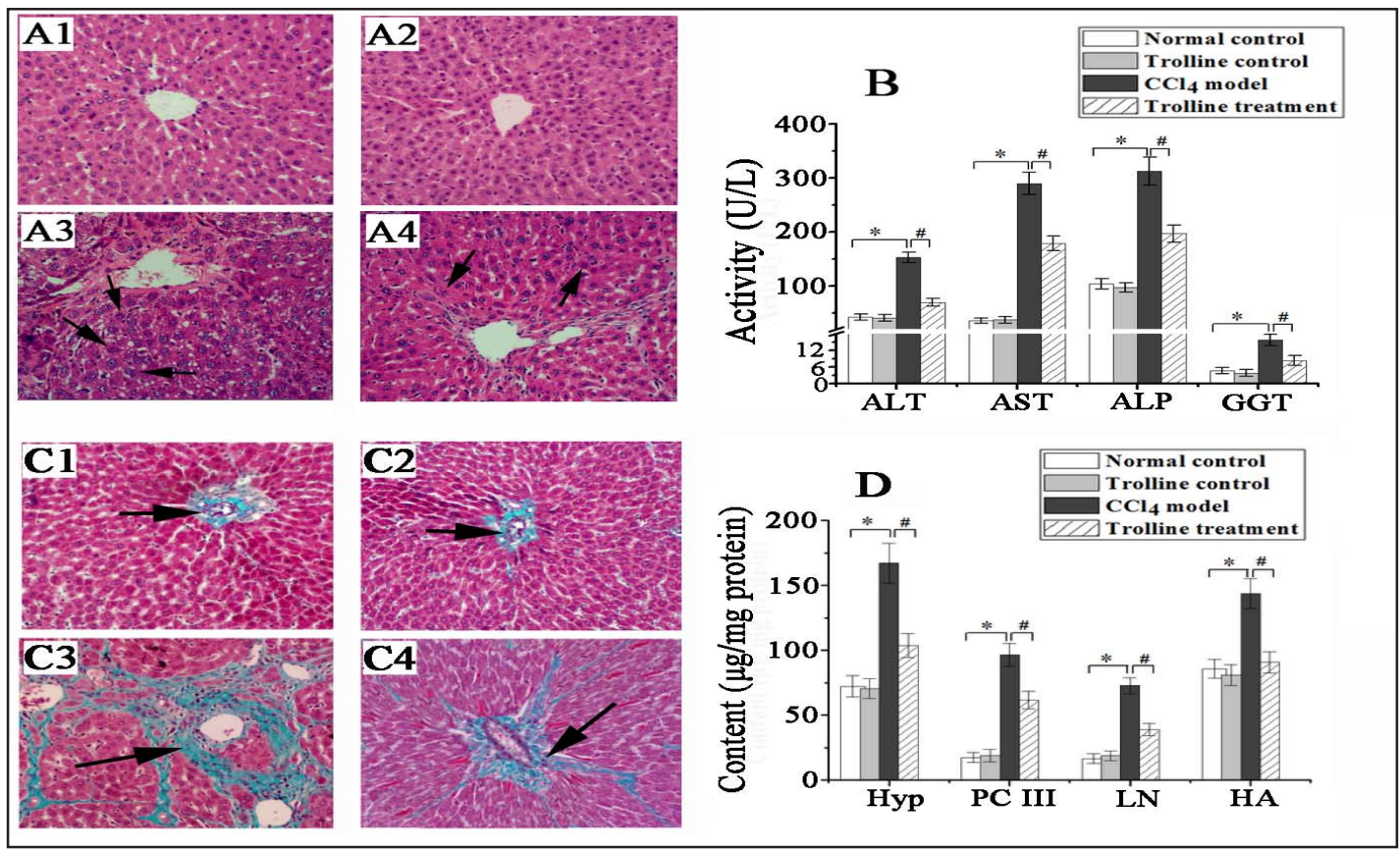

Fig. 2. Trolline significantly alleviated liver injury and collagen accumulation induced by $\mathrm{CCl}_{4}$ in rats. (A) Hepatic histological changes were observed by Hematoxylin and eosin (H\&E) staining (200 ×); A1 to A4 represent the normal control, trolline control, $\mathrm{CCl}_{4}$ model and trolline-treated groups, respectively. Arrows indicate necrosis. (B) ALT, AST, ALP and GGT activity was detected using commercially available kits. (C) Hepatic collagen was observed by Masson's trichrome staining $(200 \times)$; C1 to C4 represent the normal control, trolline control, $\mathrm{CCl}_{4}$ model and trolline-treated groups, respectively. Arrows suggest collagen accumulation. (D) Hydroxyproline (Hyp), type III precollagen (PCIII), laminin (LN) and hyaluronic acid (HA) were detected using commercially available kits. Data were expressed as the means $\pm \mathrm{SD},{ }^{*} \mathrm{P}<0.05$ vs. the normal control group and ${ }^{\#} \mathrm{P}<0.05$ vs. the $\mathrm{CCl}_{4}$ model group.

\section{Results}

Trolline alleviated liver injury in rats

Hepatic injury was observed by H\&E staining. As shown in Fig. 2A, the liver tissues in the normal control and trolline control groups showed normal lobular architecture with central veins and radiating hepatic cords (Fig. $2 \mathrm{~A} 1$ and A2). The livers in the $\mathrm{CCl}_{4}$ model group revealed severe pathological damage, as evidenced by hepatocyte loosening, fatty degeneration, portal inflammation and necrosis (Fig. 2 A3); interestingly, these damaged areas were markedly reduced by trolline treatment (Fig. 2 A4). In addition, serum ALT, AST, ALP and GGT, which are biochemical indicators of hepatic damage, were significantly increased in the $\mathrm{CCl}_{4}$ model group, while trolline treatment markedly decreased the levels of ALT, AST, ALP and GGT (Fig. 2B). These results suggest that trolline can alleviate liver injury induced by $\mathrm{CCl}_{4}$ in rats.

\section{Trolline reduced fibrogenesis}

The degree of hepatic fibrosis was assessed by Masson's trichrome staining. Liver tissue in the normal control and trolline control groups showed traces of collagen only in the walls of major blood vessels (Fig. $2 \mathrm{C} 1$ and C2). Exposure to $\mathrm{CCl}_{4}$ resulted in the extensive accumulation of collagen in liver tissue (Fig. 2 C3). In contrast, trolline treatment significantly reduced collagen production and deposition (Fig. 2 C4). Moreover, the collagenic biomarkers including Hyp, PCIII, LN and HA were elevated in $\mathrm{CCl}_{4}$-treated rats. However, these elevated levels of collagenic biomarkers were significantly decreased by trolline treatment (Fig. 2D). These results indicate that trolline can significantly inhibit hepatic fibrogenesis. 


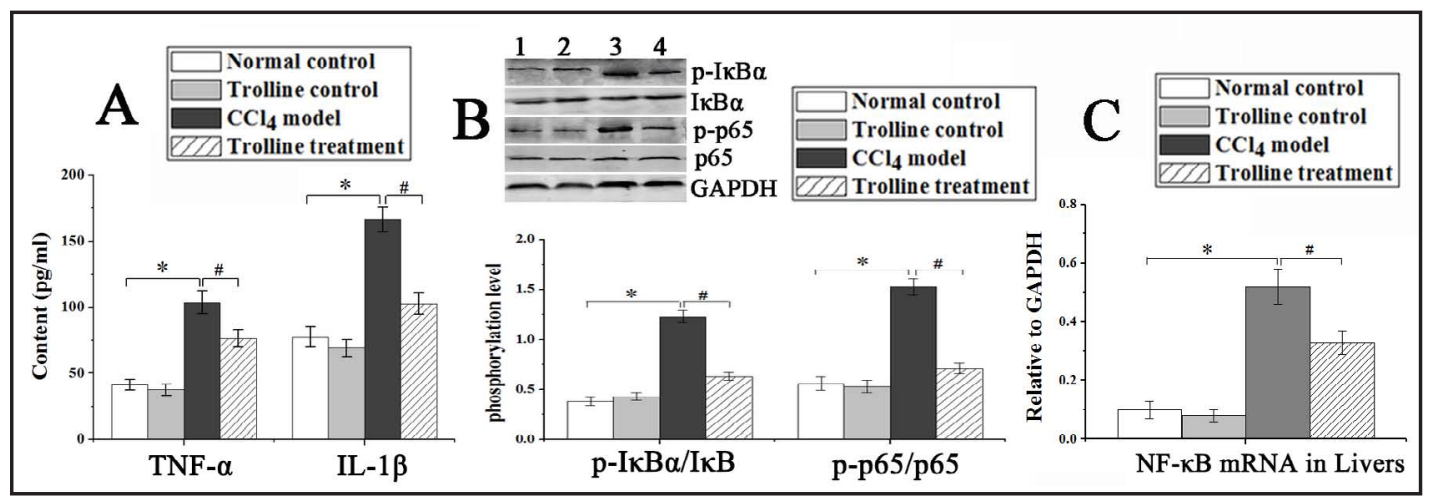

Fig. 3. Trolline significantly reduced inflammatory cytokine content and inhibited NF- $\mathrm{B}$ activation in rats. (A) The inflammatory cytokines TNF- $\alpha$ and IL-1 $\beta$ were detected by ELISA kits. (B) Phosphorylation of I $\mathrm{B} \alpha$ and p65 was detected by Western blotting; the bands 1 to 4 represent the normal control, trolline control, $\mathrm{CCl}_{4}$ model and trolline-treated groups, respectively. (C) The level of NF- $\kappa \mathrm{B}$ mRNA was detected by RT-PCR assay. Data were expressed as the means $\pm \mathrm{SD},{ }^{*} \mathrm{P}<0.05$ vs. the normal control group and ${ }^{\#} \mathrm{P}<0.05$ vs. the $\mathrm{CCl}_{4}$ model group.

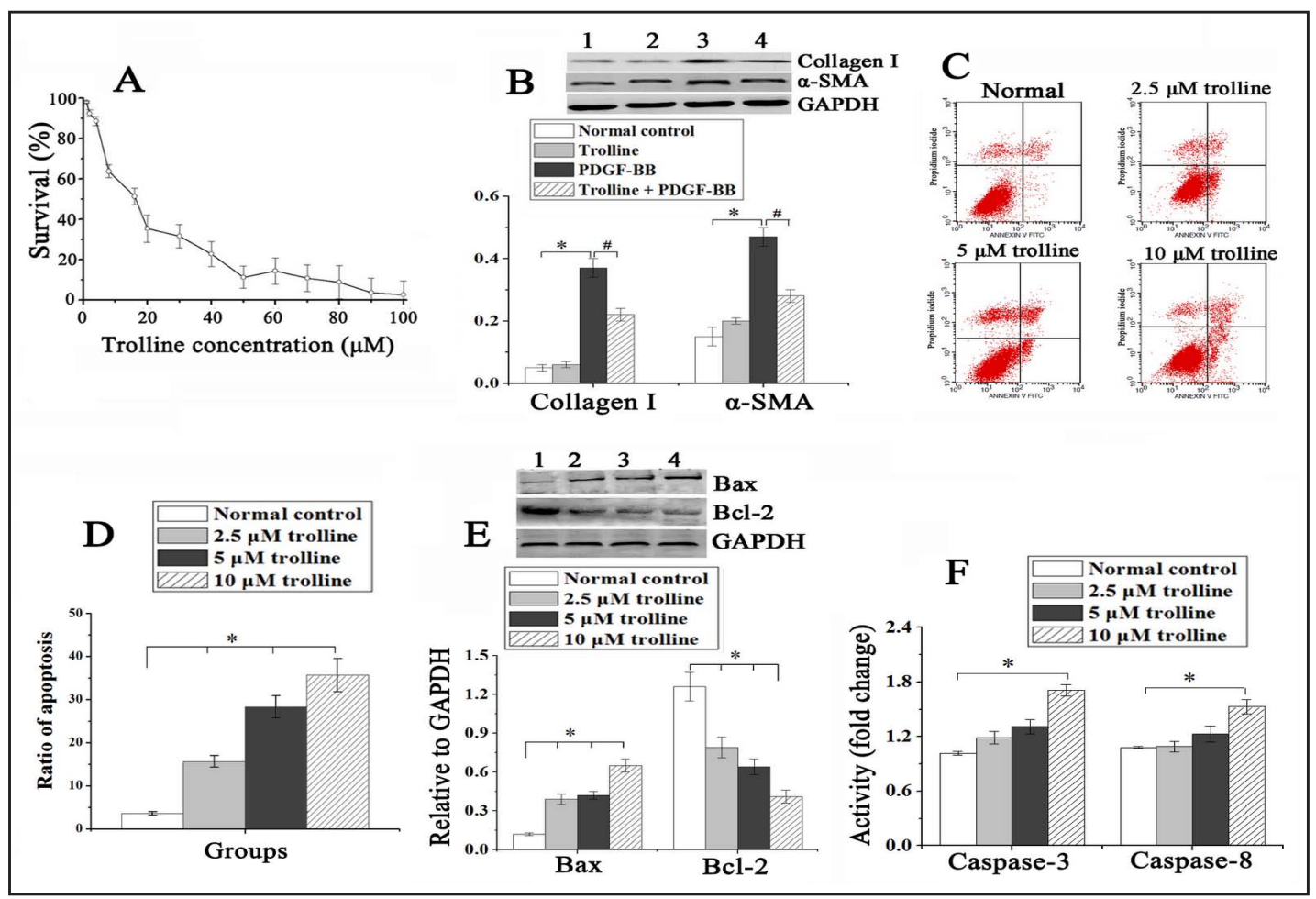

Fig. 4. Trolline significantly inhibited HSC activation and induced HSC apoptosis. (A) Cytotoxicity test was assessed by MTT assay. (B) The protein expression of $\alpha$-SMA and collagen I in HSCs was detected using Western blotting; bands 1 to 4 represent the normal tontrol, trolline control, PDGF-BB, and trolline + PDGFBB groups, respectively. (C) Cell apoptosis was assessed by flow cytometry. (D) The ratio of apoptosis was calculated. (E) The expression of Bax and Bcl-2 was detected by Western blotting; the bands 1 to 4 represent the normal control, 2.5, 5 and $10 \mu \mathrm{M}$ trolline groups, respectively. (F) Caspase-3 and -8 were detected using commercially available kits. Data were expressed as the means $\pm \mathrm{SD},{ }^{*} \mathrm{P}<0.05$ vs. the normal control group, ${ }^{\#} \mathrm{P}<0.05$ vs. the PDGF-BB group.

\section{Trolline inhibited inflammatory cytokines and NF- $\kappa B$ pathway}

The productions of TNF- $\alpha$ and IL- $1 \beta$ were significantly higher in the $\mathrm{CCl}_{4}$ model group than those in the normal control group, while the abnormal levels of both inflammatory 


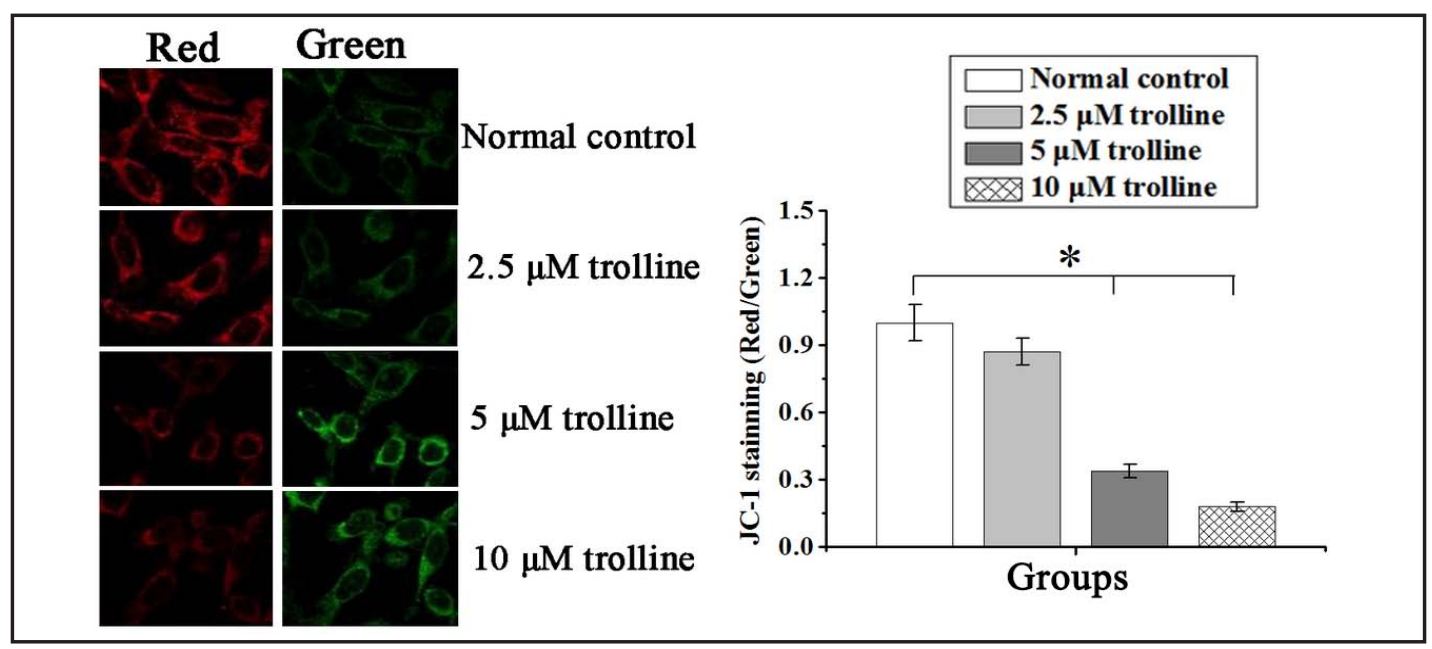

Fig. 5. Trolline significantly decreased mitochondrial membrane potential (MMP) in HSC-T6 cells. Red fluorescence represents the mitochondrial aggregate form of JC-1, indicating intact MMP; green fluorescence represents the monomeric form of JC-1, indicating MMP dissipation. Data were expressed as the means \pm SD, ${ }^{*} \mathrm{P}<0.05$ vs. the normal control group.

cytokines were reversed by trolline treatment (Fig. $3 \mathrm{~A}$ ). NF- $\mathrm{kB}$ is a key transcriptional factor for regulation of the expression of various pro-inflammatory mediators. As shown in Fig. 3B, trolline administration significantly decreased the phosphorylation of IкB $\alpha$ and p65 compared to the $\mathrm{CCl}_{4}$ model group. Similarly, the RT-PCR analysis showed that trolline inhibited the level of NF- $\mathrm{BB}$ mRNA in liver tissue (Fig. $3 \mathrm{C}$ ). These results suggest that trolline inhibits the inflammatory response via downregulation of NF- $\kappa \mathrm{B}$ activation.

\section{Trolline reduced HSC-T6 cell viability and activation}

The cytotoxicity of trolline in HSCs cells was assessed by an MTT assay. As shown in Fig. $4 \mathrm{~A}$, treatment with trolline for $24 \mathrm{~h}$ significantly inhibited cell viability in a dose-dependent manner. The $50 \%$ inhibitory concentration $\left(\mathrm{IC}_{50}\right.$ ) was $16.5 \pm 4.2 \mu \mathrm{M}$. In addition, the results showed that trolline significantly reduced the expression of $\alpha$-SMA and collagen I (Fig. 4B), suggesting that trolline can inhibit HSC activation.

\section{Trolline induced HSC apoptosis}

As shown in Fig. 4C and 4D, trolline significantly increased the ratio of apoptosis in a concentration-dependent manner in HSC-T6 cells. Moreover, trolline significantly reduced the expression of the anti-apoptotic protein Bcl-2, while increased the expression of the pro-apoptotic protein Bax (Fig. 4E). In addition, trolline markedly increased the activity of caspase-3 and -8 in a concentration-dependent manner in HSC-T6 cells (Fig. 4 F). These results indicate that trolline significantly induces HSC apoptosis partially through the modulation of the apoptosis-related protein expression and the caspase activity.

\section{Trolline reduced mitochondrial membrane potential (MMP)}

To investigate the effect of trolline on the mitochondrial pathway of cell death, MMP was assessed using JC-1 fluorescence staining. As shown in Fig. 5, trolline treatment significantly decreased red fluorescence intensity and increased green fluorescence intensity. In other words, trolline reduced MMP, therefore inducing mitochondrial dysfunction.

\section{Trolline inhibited autophagy}

Autophagy can exacerbate liver fibrosis through the degradation of lipid droplets that provide energy to HSCs. By using the autofluorescent monodansyl cadaverine dye, we observed that trolline significantly reduced the formation of autophagic vacuoles in 


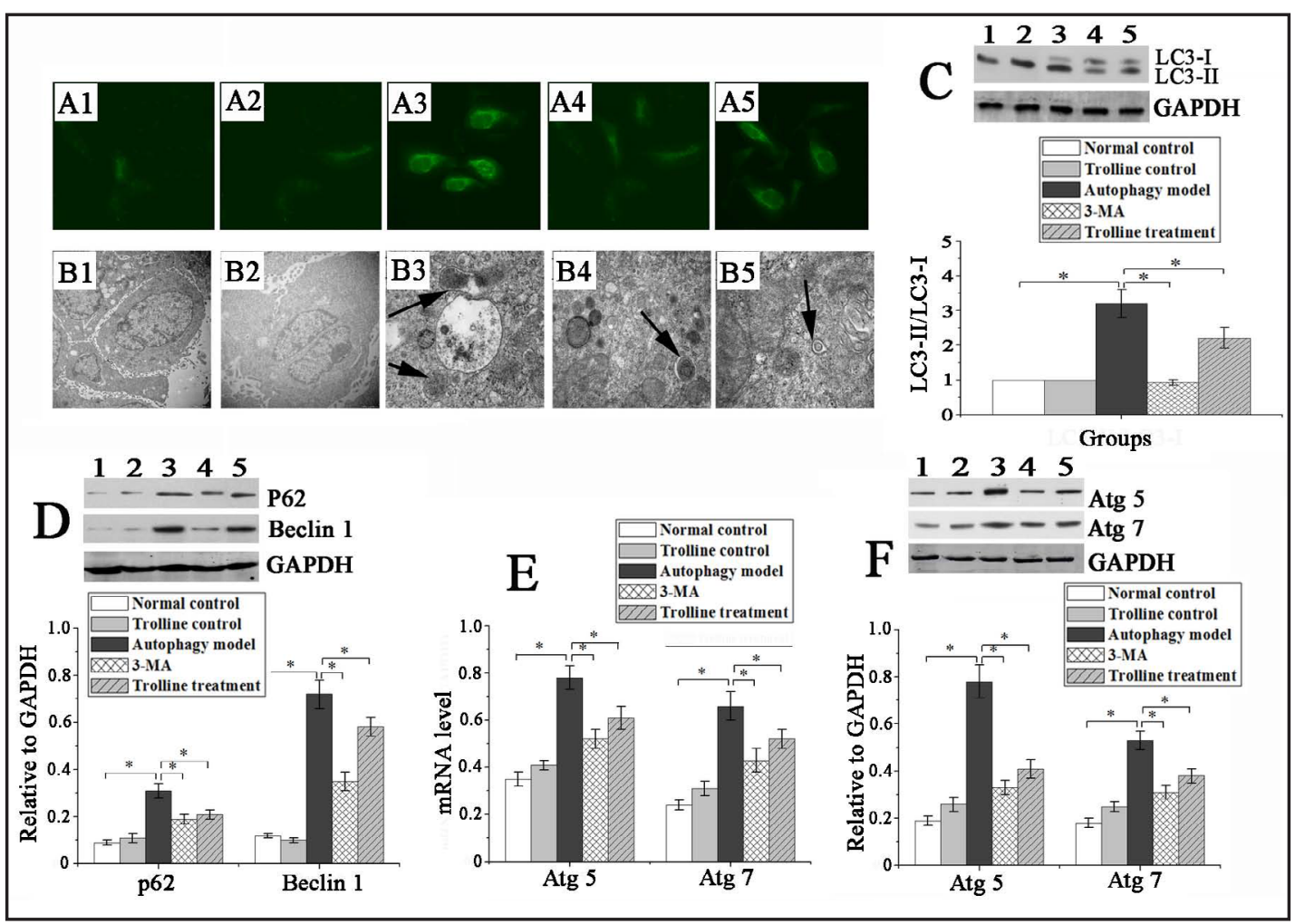

Fig. 6. Trolline significantly inhibited autophagy in HSC-T6 cells. (A) Autophagic vacuoles in HSCs-T6 cells were observed by autofluorescent monodansyl cadaverine dye; A1 to A5 represent the normal control, trolline control, autophagy model, 3-MA treatment and trolline treatment groups, respectively. (B) The autophagosomes were observed by electron microscopy; B1 to B5 represent the normal control, trolline control, autophagy model, 3-MA treatment and trolline treatment groups, respectively. (C, D and F) The proteins including LC3-I/II, P62, Beclin-1, Atg5 and Atg7 were detected by Western blotting; the bands 1to 5 represent the normal control, trolline control, autophagy model, 3-MA treatment and trolline treatment groups, respectively. (E) The mRNA expression of Atg5 and Atg7 was detected by RT-PCR. Data were expressed as the means $\pm \mathrm{SD},{ }^{*} \mathrm{P}<0.05$ vs. the autophagy model group.

HSCs-T6 cells compared to the model control group (Fig. 6 A). Moreover, we used electron microscopy to observe autophagosomes. Compared with the normal control group, increased lysosomes and autophagosomes were observed in the autophagy model group. As expected, following trolline treatment, cell structure showed more integrity, with fewer lysosomes and autophagosomes (Fig. 6 B). In addition, we also analyzed the important markers of autophagosome formation, such as Beclin-1, LC3, and P62. As shown in Fig. 6 C and $\mathrm{D}$, compared to the autophagy model group, trolline notably decreased the ratio of LC3II/LC3-I, and the expression levels of Beclin-1 and P62. To further confirm our findings, we also analyzed the levels of Atg 5 and 7, which are involved in conversion LC3-I to LC3-II. As shown in Fig. $6 \mathrm{E}$ and F, trolline significantly reduced both mRNA and protein expressions of Atg 5 and 7. These results indicate that trolline attenuates liver fibrosis, at least in part, by inhibiting autophagy in HSCs.

\section{Discussion}

Liver fibrosis, a transient process that occurs before the end-stage of cirrhosis, is caused mainly by chronic liver injury [20,21]. In this study, hepatic fibrosis model was induced by $\mathrm{CCl}_{4}$ in rats. The $\mathrm{H} \& \mathrm{E}$ staining showed that $\mathrm{CCl}_{4}$ administration led to severe histological damage 
of liver tissue; however, trolline treatment significantly attenuated the degree of liver injury. Moreover, the serological assay revealed a significant increase in the activity of serum ALT, AST, ALP and GGT in the $\mathrm{CCl}_{4}$ model group, indicating serious hepatocellular injury; while trolline treatment notably decreased the activity of these enzymes. Furthermore, our study showed that $\mathrm{CCl}_{4}$ caused extensive accumulation of collagen and increased the contents of the collagen-related indicators Hyp, PCIII, LN and HA; however, these abnormal changes in collagen induced by $\mathrm{CCl}_{4}$ were reversed by trolline treatment. These results indicate that trolline treatment can alleviate $\mathrm{CCl}_{4}$-induecd liver injury and fibrosis in rats.

Inflammation is an important pathological mechanism that propagates liver injury. As an essential processe for subsequent fibrogenesis, the inflammatory response is known to participate in collagen synthesis and accumulation. Therefore, decreasing the levels of proinflammatory cytokines may be beneficial in liver fibrosis. In the present study, the contents of IL- $1 \beta$ and TNF- $\alpha$ were significantly increased in the $\mathrm{CCl}_{4}$ model group but were markedly decreased in the trolline-treated rats, suggesting that trolline ameliorates liver injury, possibly through restricting the levels of inflammatory cytokines. To further investigate the underlying mechanism of trolline against inflammation, we also examined the activation of the NF- $\mathrm{BB}$ pathway, which is critical for the expression of various pro-inflammatory enzymes and cytokines. Its activation occurs mainly through phosphorylation and thereby

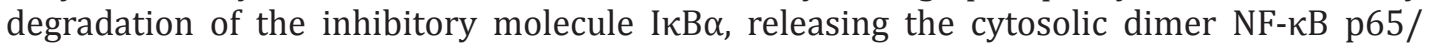
p50. The cytosolic dimer translocates into the nucleus and binds to DNA to stimulate the transcription of target genes [22]. Our results showed that $\mathrm{CCl}_{4}$ dramatically induced NF- $\mathrm{BB}$ activation as evidenced by the increase in the phosphorylation of IкB $\alpha$ and p65. However, trolline treatment significantly decreased $\mathrm{CCl}_{4}$-induced NF- $\mathrm{KB}$ activation. Taken together, these results suggest that trolline attenuates inflammation in the liver, at least in part, by suppressing the NF- $\kappa$ B signaling pathway.

Hepatic stellate cells (HSCs), the primary effector cells in the liver, are involved in the development of pathological fibrosis $[23,24]$. Thus, insight into mechanisms that regulate HSC activation is considered key for the treatment of hepatic fibrosis. In this study, trolline treatment significantly reduced HSC-T6 cell viability, as shown in the MTT assay, and inhibited HSC-T6 activation, as evidenced by the decrease in expression of $\alpha$-SMA and collagen I. On the other hand, apoptosis plays an important role in cell proliferation, differentiation, senescence and death. The potential to induce HSC apoptosis has also become an important strategy in the treatment of liver fibrosis $[25,26]$. In the present study, our results firstly showed that trolline markedly promoted HSC-T6 cell apoptosis. To further examine the role of trolline in cell apoptosis, apoptosis-related proteins (Bcl-2 and Bax), caspase activity (caspases-3 and -8), and mitochondria membrane potential (MMP) were also assessed in HSC-T6 cells. The Bcl-2 family protein is comprised of pro-apoptotic proteins (e.g. Bax) and anti-apoptotic proteins (e.g. Bcl-2). The ratio of Bax to Bcl-2 plays an important role in determining whether cells will undergo death or survival [27]. Meanwhile, caspase activation is the molecular hallmark of apoptosis and plays an important role in apoptosis [28]. Additionally, previous studies have confirmed that mitochondria play a pivotal role in cell metabolism and cell survival. Main pathways of cell death are activated in response to mitochondrial dysfunction. Moderate injuries cause the collapse of mitochondria membrane potential (MMP) and lead to an increase of pores in the mitochondrial outer membrane, resulting in cytochrome $\mathrm{c}$ release into the cytosol and potentially leading to apoptosis [29]. In the present study, we found that trolline significantly increased the ratio of Bax/Bcl-2 and the activity of caspase- 3 and -8 , while decreasing MMP, suggesting that trolline significantly induces HSC-T6 cell apoptosis via modulation of apoptosis-related protein expression, caspase activity and MMP level. Taken together, our results indicate that trolline exerts anti-hepatic fibrosis, largely through inhibiting HSC activation and promoting cell apoptosis.

Autophagy, a process by which cells degrade and metabolize their own constituents, is an evolutionary conserved fundamental cellular process. It is mediated by several autophagyrelated proteins, such as LC3, Beclin-1 and P62. Activation of autophagy is accompanied by transformation of LC3-I into LC3-II, as well as P62 degradation [30]. Recently, it was 


\section{Cellular Physiology Cell Physiol Biochem 2017;44:436-446 \begin{tabular}{c|c|c|} 
DOI: 10.1159/000485009 & $\begin{array}{l}\text { O 2017 The Author(s). Published by S. Karger AG, Basel } \\
\text { www.karger.com/cpb }\end{array}$ \\
\hline
\end{tabular} \\ Bai et al.: Trolline Ameliorates Liver Fibrosis}

reported that autophagy exacerbated liver fibrosis through the degradation of lipid droplets in HSCs providing energy for the activation of HSCs. In contrast, inhibiting autophagy could decrease HSC proliferation and promote HSC apoptosis [31], suggesting that autophagy may be a possible target in the prevention of HSC activation. In this study, we found that trolline significantly reduced the formation of autophagic vacuoles and the number of autophagosomes. Moreover, it markedly inhibited the conversion of LC3-I to LC3-II through decreasing the expression levels of Atg 5 and 7. Additionally, trolline markedly decreased the expression of Beclin-1 and P62. Our findings indicate that trolline may inhibit the activation of HSCs partially through down-regulation of autophagy.

\section{Conclusion}

Our results indicate that trolline significantly ameliorates hepatic fibrosis by inhibiting the NF- $\kappa \mathrm{B}$ pathway, promoting HSC apoptosis, and suppressing HSC autophagy. This study demonstrates that trolline has great potential as a new agent for the treatment of liver fibrosis.

\section{Disclosure Statement}

The authors declare that there are no conflicts of interest.

\section{Acknowledgements}

The authors gratefully acknowledge the financial support provided by the National Natural Science Foundation of China (No.81473431; No.81660693; No.81660686; No.81660706) and the Guangxi Natural Science Foundation (2016GXNSFDA380025).

\section{References}

1 Wynn T: Cellular and molecular mechanisms of fibrosis. J Pathol 2008;214:199-210.

2 Huang Q Liang C, Wei L, Nie J, Lu S, Lu C, Zhuo L, Lu Z, Lin X: Raf Kinase Inhibitory Protein DownExpression Exacerbates Hepatic Fibrosis In vivo and In vitro. Cell Physiol Biochem 2016;40:49-61.

-3 Kisseleva T, Brenner DA: Role of hepatic stellate cells in fibrogenesis and the reversal of fibrosis. J Gastroenterol Hepatol 2007;22:S73-S78.

4 Kim MJ, Park SA, Kim CH, Park SY, Kim JS, Kim DK, Nam JS, Sheen YY: TGF-beta Type I Receptor Kinase Inhibitor EW-7197 Suppresses Cholestatic Liver Fibrosis by Inhibiting HIF1alpha-Induced Epithelial Mesenchymal Transition. Cell Physiol Biochem 2016;38:571-588.

5 Fallowfield JA: Therapeutic targets in liver fibrosis. Am J Physiol Gastrointest Liver Physiol 2011;300:G709-G715.

\6 Oakley F, Meso M, Iredale JP, Green K, Marek CJ, Zhou X, May MJ, Millward-Sadler H, Wright MC, Mann DA: Inhibition of inhibitor of $\kappa \mathrm{B}$ kinases stimulates hepatic stellate cell apoptosis and accelerated recovery from rat liver fibrosis. Gastroenterology 2005;128:108-120.

7 Elsharkawy AM, Mann DA: Nuclear factor- $\kappa$ B and the hepatic inflammation-fibrosis-cancer axis. Hepatology 2007;46:590-597.

8 Booth LA, Tavallai S, Hamed HA, Cruickshanks N, Dent P: The role of cell signalling in the crosstalk between autophagy and apoptosis. Cell Signal 2014;26:549-555.

$>9$ Thoen LF, Guimarães EL, Dollé L, Mannaerts I, Najimi M, Sokal E, van Grunsven LA: A role for autophagy during hepatic stellate cell activation. J Hepatol 2011;55:1353-1360. 


\section{Cellular Physiology Cell Physiol Biochem 2017;44:436-446 \begin{tabular}{l|l|l} 
DOI: 10.1159/000485009 & $\begin{array}{l}\text { O 2017 The Author(s). Published by S. Karger AG, Basel } \\
\text { wwww.karger.com/cpb }\end{array}$
\end{tabular}

10 Hernández-Gea V, Ghiassi-Nejad Z, Rozenfeld R, Gordon R, Fiel MI, Yue Z, Czaja MJ, Friedman SL: Autophagy releases lipid that promotes fibrogenesis by activated hepatic stellate cells in mice and in human tissues. Gastroenterology 2012;142:938-946.

11 Li Y-L, Ma S-C, Yang Y-T, Ye S-M, But PP-H: Antiviral activities of flavonoids and organic acid from Trollius chinensis Bunge. J Ethnopharmacol 2002;79:365-368.

12 Wang RF, Yang XW, Ma CM, Cai SQ Li JN, Shoyama Y: A bioactive alkaloid from the flowers of Trollius chinensis. Heterocycles 2004;63:1443-1448.

13 Kanemitsu T, Yamashita Y, Nagata K, Itoh T: Synthesis of (-)-Trolline,(-)-Crispin A and (-)-Crispine E. Heterocycles 2008;74:199-204.

14 Lin X, Bai F, Nie J, Lu S, Lu C, Zhu X, Wei J, Lu Z, Huang Q: Didymin Alleviates Hepatic Fibrosis Through Inhibiting ERK and PI3K/Akt Pathways via Regulation of Raf Kinase Inhibitor Protein. Cell Physiol Biochem 2016;40:1422-1432.

15 Lin X, Chen Y, Lv S, Tan S, Zhang S, Huang R, Zhuo L, Liang S, Lu Z, Huang Q: Gypsophila elegans isoorientin attenuates $\mathrm{CCl}_{4}$-induced hepatic fibrosis in rats via modulation of NF- $\kappa \mathrm{B}$ and TGF- $\beta 1 /$ Smad signaling pathways. Int Immunopharmacol 2015;28:305-312.

16 Wei J, Huang Q Bai F, Lin J, Nie J, Lu S, Lu C, Huang R, Lu Z, Lin X: Didymin induces apoptosis through mitochondrial dysfunction and up-regulation of RKIP in human hepatoma cells. Chem Biol Interact 2017;261:118-126.

17 Biederbick A, Kern HF, Elsasser HP: Monodansylcadaverine (MDC) is a specific in vivo marker for autophagic vacuoles. Eur J Cell Biol 1995;66:3-14.

18 Wang Y, Gao J, Zhang D, Zhang J, Ma J, Jiang H: New insights into the antifibrotic effects of sorafenib on hepatic stellate cells and liver fibrosis. J Hepatol 2010;53:132-144.

19 Lin X, Wei J, Chen Y, He P, Lin J, Tan S, Nie J, Lu S, He M, Lu Z, Huang Q: Isoorientin from Gypsophila elegans induces apoptosis in liver cancer cells via mitochondrial-mediated pathway. J Ethnopharmacol 2016;187:187-194.

20 Li B, Cong M, Zhu Y, Xiong Y, Jin W, Wan Y, Zhou Y, Ao Y, Wang H: Indole-3-Carbinol Induces Apoptosis of Hepatic Stellate Cells through K63 De-Ubiquitination of RIP1 in Rats. Cell Physiol Biochem 2017;41:14811490.

21 Wei Y, Huang M, Liu X, Yuan Z, Peng Y, Huang Z, Duan X, Zhao T: Anti-fibrotic effect of plumbagin on CCl(4)lesioned rats. Cell Physiol Biochem 2015;35:1599-1608.

22 Hellerbrand C, Jobin C, Licato L, Sartor R, Brenner D: Cytokines induce NF- $\kappa$ B in activated but not in quiescent rat hepatic stellate cells. Am J Physiol Gastrointest Liver Physiol 1998;275:G269-G278.

23 Yu F, Guo Y, Chen B, Shi L, Dong P, Zhou M, Zheng J: LincRNA-p21 Inhibits the Wnt/beta-Catenin Pathway in Activated Hepatic Stellate Cells via Sponging MicroRNA-17-5p. Cell Physiol Biochem 2017;41:1970-1980.

24 Yu F, Yang J, Huang K, Pan X, Chen B, Dong P, Zheng J: The Epigenetically-Regulated microRNA-378a Targets TGF-beta2 in TGF-beta1-Treated Hepatic Stellate Cells. Cell Physiol Biochem 2016;40:183-194.

25 Jin S, Li H, Han M, Ruan M, Liu Z, Zhang F, Zhang C, Choi Y, Liu B: Mesenchymal Stem Cells with Enhanced Bcl-2 Expression Promote Liver Recovery in a Rat Model of Hepatic Cirrhosis. Cell Physiol Biochem 2016;40:1117-1128.

-26 Li G, Li J, Li C, Qi H, Dong P, Zheng J, Yu F: MicroRNA-125a-5p Contributes to Hepatic Stellate Cell Activation through Targeting FIH1 Cell Physiol Biochem 2016;38:1544-1552.

27 Khan N, Afaq F, Mukhtar H: Apoptosis by dietary factors: the suicide solution for delaying cancer growth. Carcinogenesis 2006;28:233-239.

28 Sun XM, MacFarlane M, Zhuang J, Wolf BB, Green DR, Cohen GM: Distinct caspase cascades are initiated in receptor-mediated and chemical-induced apoptosis. J Biol Chem 1999;274:5053-5060.

29 Wu W, Zhou X, Liu P, Fei W, Li L, Yun H: Isoflurane reduces hypoxia/reoxygenation-induced apoptosis and mitochondrial permeability transition in rat primary cultured cardiocytes. BMC Anesthesiol 2014;10.1186/1471-2253-14-1714-17.

30 Gracia-Sancho J, Guixe-Muntet S, Hide D, Bosch J: Modulation of autophagy for the treatment of liver diseases. Expert Opin Investig Drugs 2014;23:965-977.

-31 Hernandez-Gea V, Ghiassi-Nejad Z, Rozenfeld R, Gordon R, Fiel MI, Yue Z, Czaja MJ, Friedman SL: Autophagy releases lipid that promotes fibrogenesis by activated hepatic stellate cells in mice and in human tissues. Gastroenterology 2012;142:938-946. 